\title{
Differential odor processing in two olfactory pathways in the honeybee
}

\author{
Nobuhiro Yamagata ${ }^{1,2+}$, Michael Schmuker ${ }^{1}$, Paul Szyszka ${ }^{3}$, Makoto Mizunami $^{2+}$ and Randolf Menzel ${ }^{*}$ \\ 1 Institut für Neurobiologie, Freie Universität Berlin, Berlin, Germany \\ 2 Graduate School of Life Sciences, Tohoku University, Sendai, Japan \\ 3 Neurobiologie, Universität Konstanz, Konstanz, Germany
}

\section{Edited by:}

Zachary F. Mainen, Cold Spring Harbor

Laboratory, USA

\section{Reviewed by:}

Hanna Mustaparta, Norwegian

University of Science and Technology,

Norway

Brian Smith, Arizona State University, USA

\section{${ }^{*}$ Correspondence:}

Randolf Menzel, Neurobiologie, Institut für Biologie, Freie Universität Berlin

Fachbereich Biologie, Chemie,

Pharmazie, Königin-Luise-Strasse

28/30, 14195 Berlin-Dahlem, Germany.

e-mail:menzel@neurobiologie.fu-

berlin.de

\section{tPresent address:}

Nobuhiro Yamagata, Centre de

Recherches sur la Cognition Animale,

CNRS UMR 5169, Université Paul

Sabatier, Toulouse Cedex 4, France;

Makoto Mizunami, Graduate School of

Life Science, Hokkaido University,

Hokkaido, Japan.
An important component in understanding central olfactory processing and coding in the insect brain relates to the characterization of the functional divisions between morphologically distinct types of projection neurons (PN). Using calcium imaging, we investigated how the identity, concentration and mixtures of odors are represented in axon terminals (boutons) of two types of PNs - IPN and mPN. In IPN boutons we found less concentration dependence, narrow tuning profiles at a high concentration, which may be optimized for fine, concentration-invariant odor discrimination. In mPN boutons, however, we found clear rising concentration dependence, broader tuning profiles at a high concentration, which may be optimized for concentration coding. In addition, we found more mixture suppression in IPNs than in mPNs, indicating IPNs better adaptation for synthetic mixture processing. These results suggest a functional division of odor processing in both PN types.

Keywords: calcium imaging, olfaction, projection neuron, mushroom body, parallel processing, functional division, insect

\section{INTRODUCTION}

Animals perceive their external chemical environment by a repertory of odorant receptor neurons (ORN), each of which expresses only one or two members of the odorant receptor gene family (Chess et al., 1994; Vosshall et al., 2000). The axons of ORNs expressing the same gene converge at a specific set of glomeruli in the primary olfactory center, the main olfactory bulb (MOB) in mammals and the antennal lobe $(\mathrm{AL})$ in insects, thus providing a map of spatially arranged functional modules (Mombaerts et al., 1996; Gao et al., 2000; Vosshall et al., 2000).

The primary olfactory center is generally segmented into several subregions. In mice, the MOB is subdivided into two nonoverlapping regions by a separation of olfactory epithelium (OE), a dorsal zone and a ventral zone, as indicated by an axonal surface glycoprotein, OCAM (Yoshihara et al., 1997). The mouse ORNs can be classified into two groups by the gene family; the class I and class II receptors, the former being exclusively expressed in the dorsal zone of the OE (Zhang et al., 2004). Moreover, the dorsal MOB is clearly subdivided by these two receptor types (Bozza et al., 2009). Similarly, the AL of the insects can be subdivided with respect to the origin of second order olfactory projection neurons (PN) that receive input from non-overlapping glomeruli (uniglomerular PN, Homberg et al., 1988; Stocker et al., 1990; Malun et al., 1993; Rø et al., 2007; Zube et al., 2008).
The topographical organization is particularly well described in the honeybee, in which four sensory tracts of ORNs subdivide the AL into four regions (T1-4; Mobbs, 1982; Abel et al., 2001; Kirschner et al., 2006; see Figure 1B). PNs that run through the lateral antenno-cerebral tract (IPN) innervate frontally located T1 glomeruli, while PNs in the medial antenno-cerebral tract (mPN) innervate proximally located T2-4 glomeruli in the AL. The central projection areas of the two PN tracts are segregated in the mushroom body (MB) calyx and the lateral horn (LH) with partial overlap (Abel et al., 2001; Kirschner et al., 2006).

Unlike other sensory systems, a topographic functional organization, i.e. 'Chemotopy', is not found in the primary olfactory centers (Fishilevich and Vosshall, 2005; Hallem and Carlson, 2006; Soucy et al., 2009), implying their zonal organizations do not relate to chemical properties of odorants. In addition, non-sexual social pheromones in honeybees are processed together with general odors in the primary olfactory center (Sandoz, 2006; Sandoz et al., 2007), in sharp contrast to sex pheromone that are processed in specialized regions (Christensen and Hildebrand, 2002; Lei et al., 2004). No functional separation or distinction between such social pheromone and general odor processing has been observed in the bees' two parallel PN pathways (Sun et al., 1993; Abel et al., 2001; Müller et al., 2002). In mice, however, it was reported that glomerular subsets of the MOB that correspond to the dorsal zone of the olfactory 
epithelium mediate innate responses to aversive odors (Kobayakawa et al., 2007). Further studies are required to understand how the brain interprets categories of odors, and uses the topographical information in the olfactory system.

Electrophysiological studies of honeybee PN suggest a functional separation of IPNs and mPNs in odor coding (Müller et al., 2002; Krofczik et al., 2009). The conclusions of these two studies are not fully consistent, and are based on a relatively small number of sampled neurons due to technical difficulties encountered in intracellular electrophysiology.

Here we used selective infiltration of $\mathrm{Ca}^{2+}$ sensors via the somata to measure odor-induced activity in boutons of the lPNs and mPNs in the MB calyces. We found (1) less concentration dependence in IPNs, but clearly rising concentration dependence in mPNs, (2) more narrow tuning profiles in IPNs and broad tuning profiles in $\mathrm{mPNs}$ at a high odor concentration, and (3) more mixture suppression in IPNs than in mPNs. These results suggest a functional division of odor processing in the two types of PN.

\section{MATERIALS AND METHODS PREPARATION AND DYE LOADING}

Foraging worker honeybees were collected at the entrance of laboratory hives, chilled, fixed in recording chambers with low temperature melting wax (Joerges et al., 1997; Szyszka et al., 2005), and fed with a drop of $30 \%$ sucrose solution. The head capsule between the compound eyes was opened. Glands and trachea sacks covering the AL were carefully removed. The tip of a pulled glass capillary was broken forming a tip diameter of $\sim 10 \mu \mathrm{m}$, and was coated with a mixture of the calcium-sensitive dye Fura-dextran (10 000 MW, Molecular Probes, Eugene, OR, USA) and the lysine fixable dye tetramethylrhodamine-dextran (10000 MW, Molecular Probes, Eugene, OR, USA) dissolved in distilled water. The dye-coated tips were used to penetrate either the soma cluster of the IPNs or mPNs. Somata of IPNs are located antero-ventral to the AL, and those of $\mathrm{mPNs}$ are located medio-postero-dorsal to the AL (Figure 1A, Abel et al., 2001; Kirschner et al., 2006). The head capsule was then closed with the cut cuticle piece and sealed with $n$-eicosan (Sigma). The bees were fed until satiation and kept in a moist container at $17-20^{\circ} \mathrm{C}$ for $8-24 \mathrm{~h}$. To stabilize the brain, the legs and wings were cut and the abdomen, thorax and mandibles were immobilized with wax. The antennae were fixed with $n$-eicosane and the calyces of the MB were exposed for measurements. All gaps around the head were sealed with vaseline (local drugstore), and the recording chambers were filled with Ringer solution (in mM: $130 \mathrm{NaCl}$, $7 \mathrm{CaCl}_{2}, 6 \mathrm{KCl}, 2 \mathrm{MgCl}_{2}, 160$ sucrose, 25 glucose and $10 \mathrm{HEPES}$, pH 6.7, 500 mosmol).

\section{ODOR STIMULATION AND IMAGING}

The odorants were diluted in decade steps to $10^{-5}$. Seven odors 1hexanol, hexanal (Merck), 1-octanol, 2-octanol, linalool, 2-heptanone, octanal (Sigma, Deisenhofen, Germany) diluted to $10^{-2}$ were used for chemo-profile experiments and three odorants hexanal, 2-heptanone, 1-octanol (at all six concentrations) were used for odor concentration experiments. Their chemical structures are shown in Figure 1C. As binary mixtures we used 1-octanol plus 2-heptanone and hexanal plus 2 -octanone ( $1 \%$ of each odor). The bees were exposed to a constant air stream, into which the head space of a syringe $(12 \mathrm{ml})$ containing a filter paper $\left(1 \mathrm{~cm}^{2} \times 2\right)$ soaked with paraffin oil (control) or one of the odorants $(40 \mu \mathrm{l})$ was injected. Injection of the control air or the odorant into the constant air stream was switched on and off by a computer-controlled solenoid valve (Galizia et al., 1997) without changing the total airflow. Odors were presented for $3 \mathrm{~s}$ three times at an interval of $90 \mathrm{~s}$.

Calcium measurements were performed at room temperature with a sampling rate of $5 \mathrm{~Hz}$, using a TILL-Photonics imaging set up mounted on a fluorescence microscope (Zeiss Axioskop, Germany). Fura was alternately excited at 340 and $380 \mathrm{~nm}$. Exposure times were $15 \sim$ and $60 \sim$ ms, respectively. Each measurement started 3 s prior to stimulus onset and lasted for $10 \mathrm{~s}$. Images were acquired through a $60 \times / 0.9$ NA, waterdip objective (Olympus, Tokyo, Japan), a 410$\mathrm{nm}$ dichroic mirror and a 440-nm long pass filter with an Imago CCD camera $(640 \times 480$ pixels, $4 \times$ binned on chip to $160 \times 120)$. Pixel size was $1.47 \times 1.47 \mu \mathrm{m}$, which allowed resolution of a single bouton of a PN.

\section{CONFOCAL MICROSCOPY}

After $\mathrm{Ca}^{2+}$ measurements, the brain was dissected and fixed in $4 \%$ formaldehyde in Millonig's buffer overnight at $4^{\circ} \mathrm{C}$ and then rinsed in saline, dehydrated in ethanol, and cleared in methyl salicylate. The brain was set into a chamber filled with methyl salicylate and observed from the frontal surface with a confocal laser-scanning microscope (Leica TCS SP2; Leica, Wetzlar, Germany). The excitation wavelength was $543 \mathrm{~nm}$ with a Green $\mathrm{HeNe}$ laser. The entire brain was scanned with a $10 \times / 0.4$ NA air objective (Olympus, Tokyo, Japan). The AL was scanned with a $20 \times / 0.70 \mathrm{NA}$ air objective (Olympus, Tokyo, Japan). The MB calyx was scanned with a $63 \times / 1.32-0.6 \mathrm{NA}$ oil objective (Olympus, Tokyo, Japan).

\section{DATA ANALYSIS}

Data were first analyzed with custom-made programs in IDL (RSI, Boulder, CO, USA). The ratio of $\mathrm{Ca}^{2+}$ signals from 340 and $380 \mathrm{~nm}$ measurements was calculated for each individual frame. Background fluorescence was determined by averaging over frames $4-13$, and the average was subtracted from every frame of the ratio metric measurements $\left(\Delta F_{340 / 380}\right)$. For visualization, these relative fluorescence changes were corrected for dye bleaching by subtracting a logarithmic curve fitted to the mean brightness decay over all image frames except for two frames at the beginning and during the stimulus (frames 15-29). A mean of 15 frames during odor stimulation was calculated and displayed as a false-color image (Figures 2, 3, 5 and 6). A spatial low-pass filter $(5 \times 5$ pixels $)$ was applied to the images for better visualization. Each morphological image was acquired as an averaged raw fluorescence image of $380 \mathrm{~nm}$ during the measurements and was later unsharp mask-filtered in Photoshop (Adobe).

Individual boutons were determined as isolated activity spots in the false-color images. For each bouton a response trace was calculated by averaging the signal of 21 pixels of an activity patch without any filtering and correction. Response traces were analyzed with Matlab (The Mathworks, Natick, MA, USA). Since individual response traces defined by such small activity patches were often noisy and could not be fitted well by logarithmic curve, they were corrected for dye bleaching by subtracting a linear regression line fitted for frames 5-14 and 40-49. An odor response (excitation/ 
inhibition) in a given bouton for a given odor was determined by comparing the response trace of an average of three trials (trial-averaged trace of the same odor) with a threshold criterion. The threshold was determined in each experiment as double the standard deviation of all fluorescence values over a period of $2 \mathrm{~s}$ before stimulation derived from trial-averaged response traces of all possible bouton/odor combinations. Specifically, an excitatory response was defined as a given time trace of the bouton exceeding the baseline amplitude (calculated as an average of pre-stimulus fluorescence values of response traces of all boutons/ odors in each bee) plus the threshold at least twice during odor application (frames 15-29). An inhibitory response was defined as the time trace being lower than the averaged amplitude minus the threshold at least twice during odor application. Since some boutons crossed both excitatory and inhibitory thresholds during odor exposures, the sum of excitatory and inhibitory responses could exceed the number of boutons (Figures 5D,E, Figure S3B in Supplementary Material).

Statistical analyses were done with Excel (Microsoft) and Matlab. Most of the statistics were performed by pooling boutons from different bees (Figures 3-6). All of the boutons for which measurements were performed were included, and either response traces of individual trials (Figure 5C, Figure S4 in Supplementary Material) or trial-averaged traces (Figures 3C, 4, 5D,E and 6, Figures S1 and S3 in Supplementary Material) were used. In order to quantify similarities in the activity of populations of boutons for each animal with respect to different concentrations of odors (Figure 3C), response traces from all boutons in the particular animal were serially concatenated into an array for each odor concentration during and after odor stimulus (frames 15-50). Similarities were then calculated by Pearson's correlation coefficient for all concentration combinations, plotted against concentration differences in log scale. Linear regression was applied for statistical tests of the relationship between them. Similarities of activity of populations of boutons with respect to different odors were calculated by Pearson's correlation coefficient applied for a series of serially concatenated response traces from all boutons for each odor (Figure 5C). Singletrial traces were used to calculate response similarity among different trials in each odor (inter-trial correlation) and compare them with those between different odors (inter-odor correlation). Due to all possible combinations of three trials, this treatment provided three inter-trial correlation coefficient values for each odor. For each odor combination, all possible combinations of three traces of two odors produced a total of nine inter-odor correlation coefficient values. These values were averaged first respectively for subsequent comparison between inter-trial and inter-odor correlations of IPN and $\mathrm{mPN}$.

To quantify odor concentration dependence of response magnitude of a given bouton (Figure 4), first the response strength of each bouton was determined for each concentration as the averaged fluorescence amplitude during the odor stimulus (trial-averaged response trace used). The relations between odor concentrations and corresponding response magnitudes in each bouton was quantified by applying linear regression (Pearson's correlation coefficient) between logarithm of rising concentrations (six steps) and of the corresponding normalized response strength (set maximum to 1 in each bouton). Boutons were then sorted into positive (rising) dependence group or negative (falling) dependence group when their response dependence was statistically significant, and sorted into the no dependence group when their dependence was not statistically significant.

In order to assess the effect of odor mixture suppression, the response strength of boutons to binary mixtures and their components were compared. If the responses to the mixture were smaller than the response to the weaker component minus noise, the bouton was assigned to the group showing mixture suppression (Figure 6). The response strength of boutons was determined in the same way described above. Noise was defined as the standard deviation of fluorescence values of the trial-averaged response traces from all boutons/odors in a bee over a period of $2 \mathrm{~s}$ before stimulation. For comparisons of the probability of the mixture suppression group between IPNs and mPNs, log-likelihood tests were applied for the absolute number of the group in pooling boutons.

\section{RESULTS}

We investigated the response properties of $1 \mathrm{PN}$ and $\mathrm{mPN}$ boutons in the lip region of the MB calyx (Figure 1). $\mathrm{PPN}$ and $\mathrm{mPN}$ s were stained with a mixture of the $\mathrm{Ca}^{2+}$ indicator Fura-dextran and the neuroanatomical tracer rhodamine-dextran via their somata. The somata of IPNs and mPNs are located separately in the rim region of the AL. IPNs were filled via an antero-ventral soma cluster, and mPNs via the medio-postero-dorsal somata cluster (Figure 1A). Combining $\mathrm{Ca}^{2+}$ imaging with subsequent anatomical evaluation allowed us to investigate the IPN and mPN boutons separately. Confocal imaging confirmed the selectiveness of the stained PN types in all bees ( 57 bees, Figure 2A) and the branching patterns of axon trees as well as their boutons in the lip region of the MB calyces (IPN: 5 bees; mPN: 6 bees, Figure 2B).

It was not possible to identify or count the number of stained glomeruli in the AL due to the extracellular dye infusions involved in dye injections. The average number of stained boutons showing excitation and/or inhibition to odor stimulation did not differ between bees stained for IPNs or mPNs (lPN: ave. \pm s.e.m., $71.7 \pm 2.9$; total, 2294, 32 bees; mPN: 77.2 \pm 4.3; total, 1931, 25 bees).

\section{ODOR RESPONSES IN PN BOUTONS}

Examples of raw fluorescence images of stained IPNs and mPNs, together with their odor responses and exemplified response traces, are shown in Figures 2C,E (left) (lPN), and 2D and 2E (right) (mPN). In most cases, boutons of PNs were visible as distinct activity spots in the images (Figures 2C,D). These spots were clearly identified as boutons of either type of $\mathrm{PN}$ by comparing the patterns of raw fluorescent images with the axon tracts and the corresponding patterns of boutons in confocal images of the same bees (Figure 2B).

Each of the morphologically identified boutons represented an isolated activity spot. As shown in Figures 2C,D, spots of odorevoked $\mathrm{Ca}^{2+}$ activity were co-localized with boutons. In some cases, activities that may have come from out-of-focus planes did not fully co-localize with the position of boutons. Since such boutons reliably responded to repeated stimuli with similar response traces, it was concluded that they also represented boutons. Figure 2E demonstrates the time course of responses to different odors/ concentrations of several boutons in both IPN and mPN. These data come from different animals, and show either excitatory or 
inhibitory responses with a whole range of time courses (10 s). The rise of fluorescent values before odor stimulation in some traces are due to either (i) spontaneous activity, and/or (ii) fluctuations of fluorescent values in the entire image at the onset of illumination due mainly to a measurement artifact.

Response traces of both IPN and mPN boutons show fast (phasic), intermediate, and slow (tonic) components. No differences, either in absolute or in relative terms, were observed between the boutons of the two tracts or between different odors (data not shown).

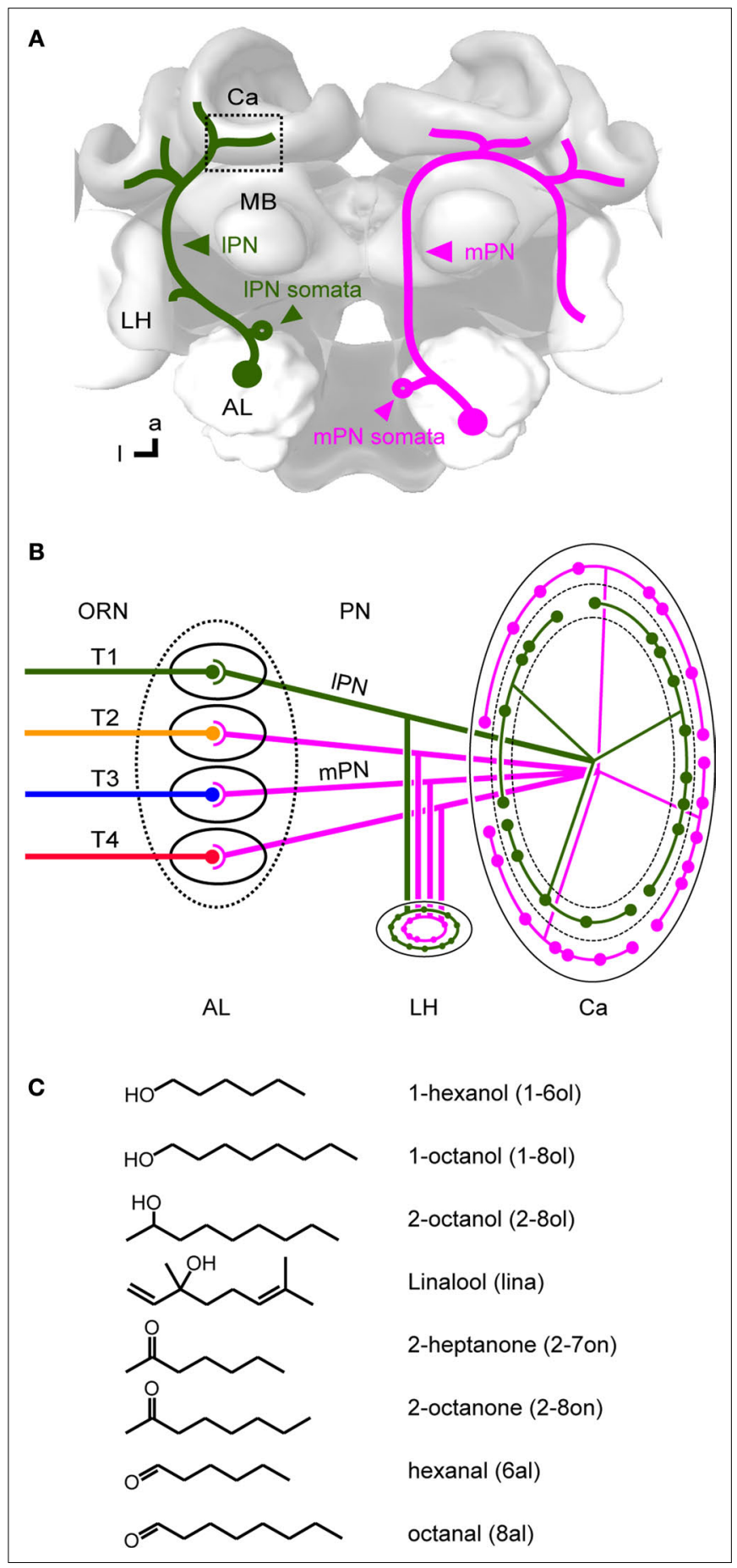

We have further analyzed individual bouton responses in some animals combined with morphological 3D reconstructions of the terminal axon branches acquired by confocal images (Figure S1 in Supplementary Material). In general, most of the boutons in an axon branch responded to odors in the same way, but some boutons from the same branch had different response profiles (see legend of Figure S1 in Supplementary Material). The observations were also supported by morphological observations in which specific co-localizations of individual PN boutons and immuno-stained GABA-profiles often occurred within an axon branch both in IPN and mPN terminal branches (Figure S2B in Supplementary Material, arrowhead). Statistical analyses revealed that responses of individual boutons in a particular axon branch are more similar than boutons in different axon branches (Figures S1B,C,D in Supplementary Material). This applies to both IPN and mPN axon branches, indicating that bouton-specific response shaping, e.g. via putative inhibitory input from GABA profiles, may act rather similarly on all boutons of the same axon branch (e.g. inhibitory input onto axon branches, Figure S1B in Supplementary Material, arrow). When comparing boutons from different axon branches we found that bouton responses are more distinct in IPNs when compared to $\mathrm{mPNs}$, indicating more specific odor and/or concentration dependence in IPN boutons (Figure S1D in Supplementary Material).

\section{DIFFERENT CONCENTRATION DEPENDENCIES IN IPN AND mPN BOUTONS}

In order to assess the representation of different concentrations of odors in IPN and mPN boutons, bees were stimulated with three different odors at six concentrations ranging over $6 \log$ units $\left(10^{-5}\right.$ to no dilution). We analyzed 1577 boutons $(71.7 \pm 3.3$, mean $\pm S D$, 22 bees) in lPNs and on 1223 boutons ( $71.9 \pm 4.8,17$ bees $)$ in mPNs. The odors hexanal (6al, lPN: 7 bees, mPN: 4 bees), 2-heptanone (2-7on, lPN: 8 bees, mPN: 8 bees) and 1-octanol (1-8ol, lPN: 7 bees, $\mathrm{mPN}$ : 5 bees) were used.

Examples of bouton responses for a concentration series of 2-7on are shown for both IPNs and mPNs (Figures 3A,B).

FIGURE 1 | Olfactory pathways in the honeybee brain. (A) Two main olfactory pathways comprised of uniglomerular projection neurons (PN) connect the primary olfactory neuropil, the antennal lobe $(A L)$, and second order neuropils, the mushroom body (MB) and the lateral horn (LH). Both types exist in each side of the brain, and are drawn here separately in the right and left side of the brain for clarity. The lateral-antennocerebral tract neurons (IPN), which travel in the protocerebrum lateral to the mushroom body, is shown in green only on the left side, and the medial-antennocerebral tract neurons (mPN), which travel in the protocerebrum medial to the MB, is shown in magenta only on the right side. These neurons were selectively stained via their somata in the AL. The square indicates the imaged region in the lip of the $M B$ calyx. (B) Wiring diagram of olfactory neurons in the $A L$ and the MB. Odors are detected by olfactory receptor neurons (ORN) on the antennae. ORN axons project into the AL via four parallel tracts (T1-4, Mobbs, 1982; Abel et al., 2001). ORNs running in the different tracts form synapses with nonoverlapping groups of glomeruli, the processing units in the AL. IPNs project from T1 glomeruli to the protocerebrum and terminate mainly in the core of the MB calyx lip (Ca). mPNs project from T2-4 glomeruli to the protocerebrum and terminate mainly in the rim of the Ca lip (Kirschner et al., 2006). Orientation of the brain is shown in neuroaxis. a: anterior and l: lateral in brain orientation. (C) Chemical structure of odors used in this study and their abbreviations. 
A
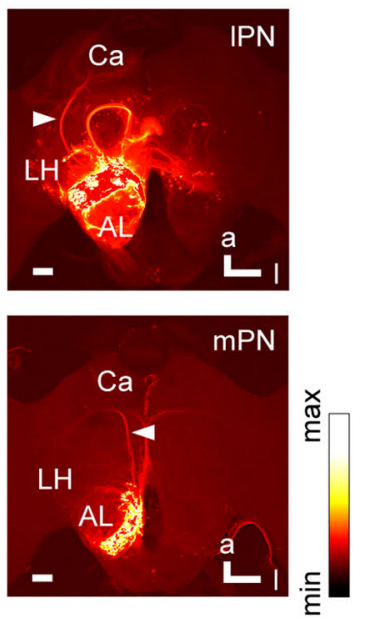

C
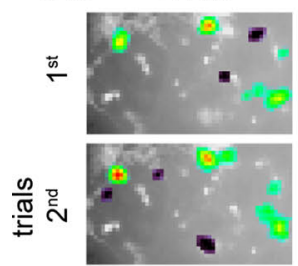

m

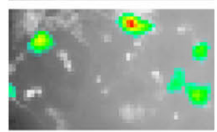

D
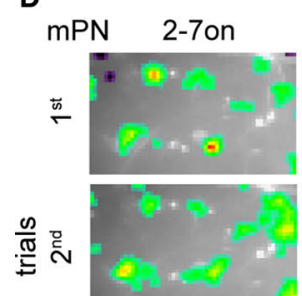

행

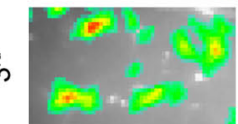

IPN 2-7on
$2-801$
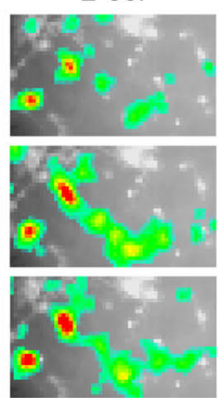

$8 a l$

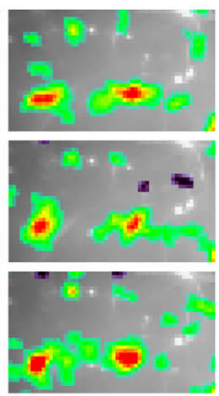

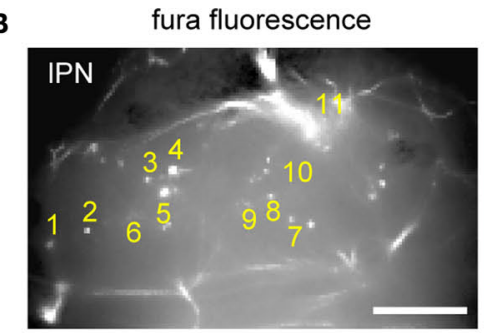
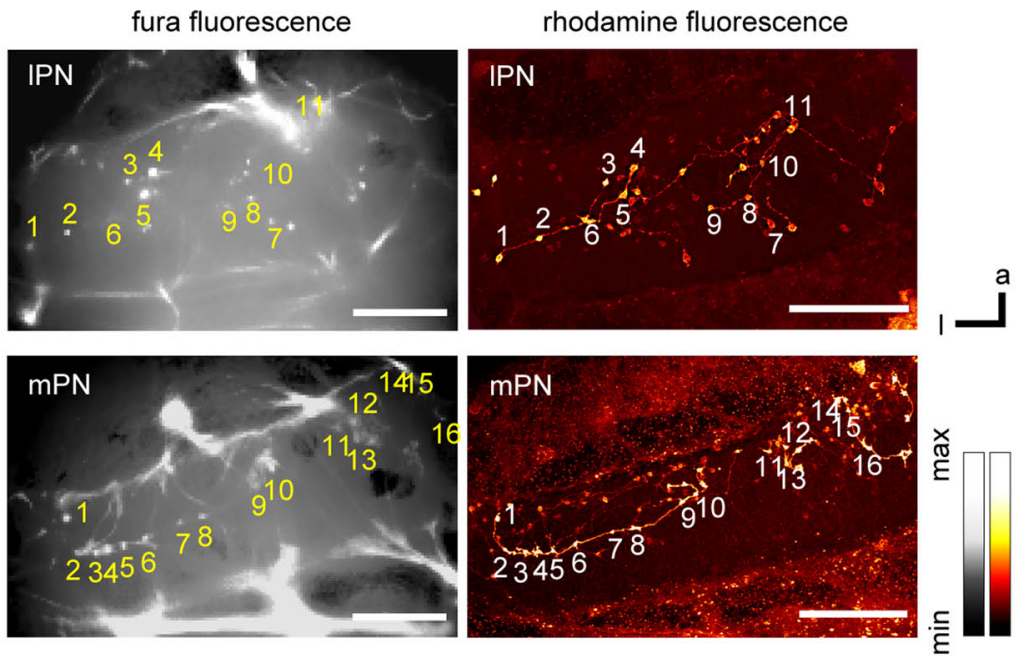

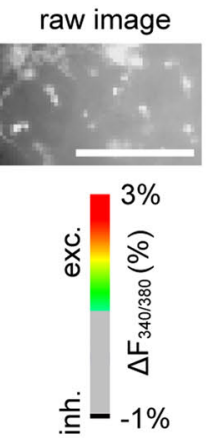

raw image

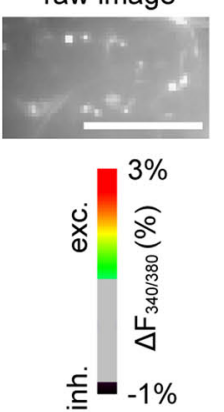

E IPN

FIGURE 2 | Dye loadings and $\mathrm{Ca}^{2+}$ signals in IPN and $\mathrm{mPN}$ boutons. (A) Confocal images confirm selective staining of IPNs or mPNs. A mixture of the calcium indicator Fura dextran and the neuro-tracer rhodamine dextran were injected into the somata clusters of the IPNs or mPNs for their selective staining. Arrowheads point to the stained tracts of PN axons. Scale bars $=100 \mu \mathrm{m}$. (B) Wide field images of Fura fluorescence (left) and confocal images of rhodamine fluorescence (right) in stained IPN and mPN boutons in the MB calyx. Tagged

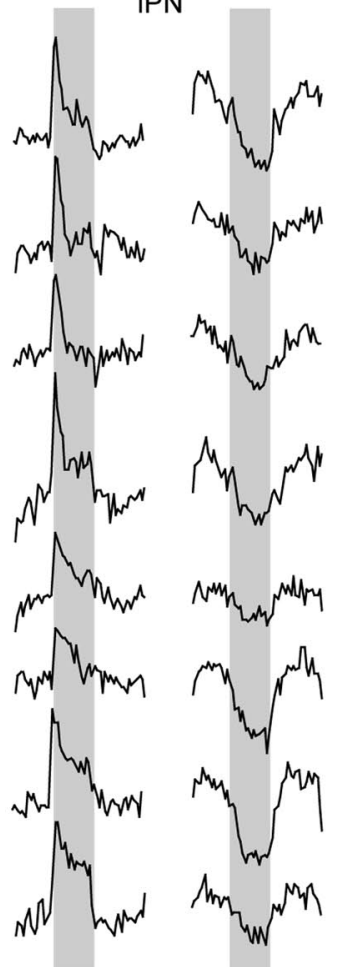

$\mathrm{mPN}$ boutons are identical between images. Scale bars $=50 \mu \mathrm{m}$. Trial-invariant odor are superimposed on color-coded $\mathrm{Ca}^{2+}$ responses of boutons. Locations of isolated activity spots and individual boutons (bright spots) match. 2-7on: 2specific $\mathrm{Ca}^{2+}$ signals in IPN (C) and mPN (D) boutons. Raw fluorescence images heptanone; 2-8ol: 2-octanol; 8al: octanal. Scale bars $=50 \mu \mathrm{m}$. (E) IPN and mPN boutons exhibited both excitatory and inhibitory responses. Response traces give $\Delta F_{340 / 380} ;$ gray bars indicate the 3s-odor stimulus. Vertical scale bar: $1 \%$ in $\Delta F_{340 / 380}$.

Both IPN and mPN boutons responded to the whole concentration range if all boutons from different bees were taken into account (Figure S3A in Supplementary Material). In both tracts, boutons exhibited reliable responses to stimulus repetitions with concentration dependent spatio-temporal response patterns.
Stimulus repetitions were reliably represented in individual boutons (see the legend of Figure S4A in Supplementary Material). We analyzed the concentration specificity of odor representations of IPN and mPN boutons in their respective bouton populations separately for each bee. The similarity of the spatio-temporal 


\section{A IPN

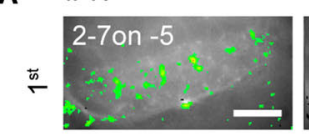

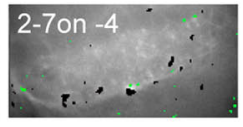
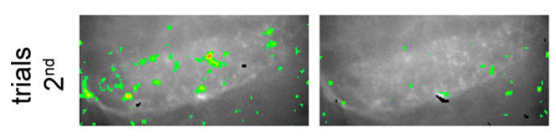

?
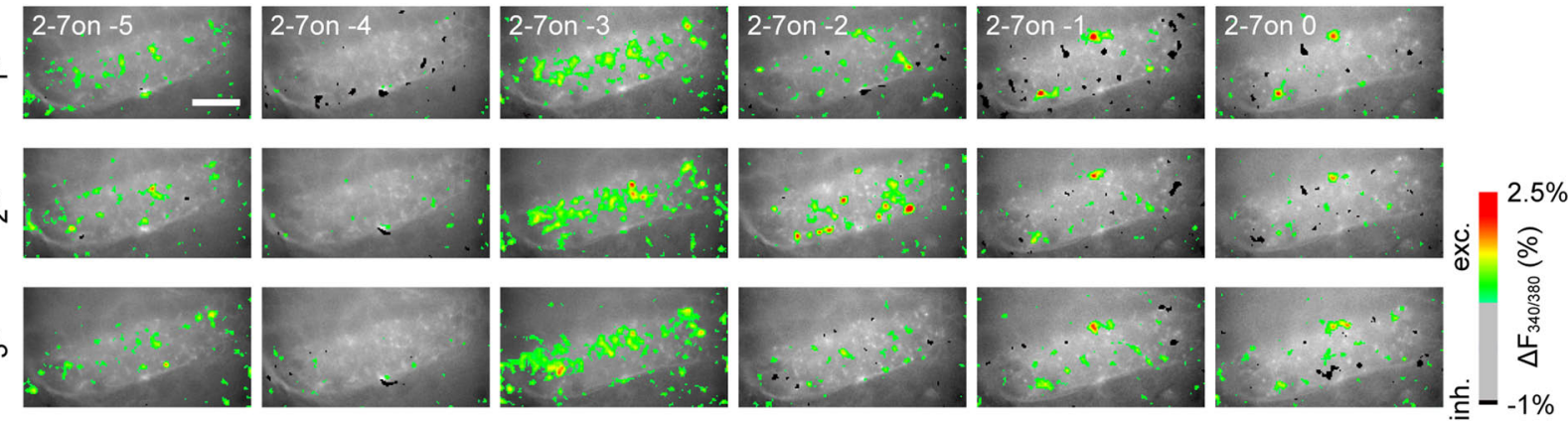

B $\mathrm{mPN}$
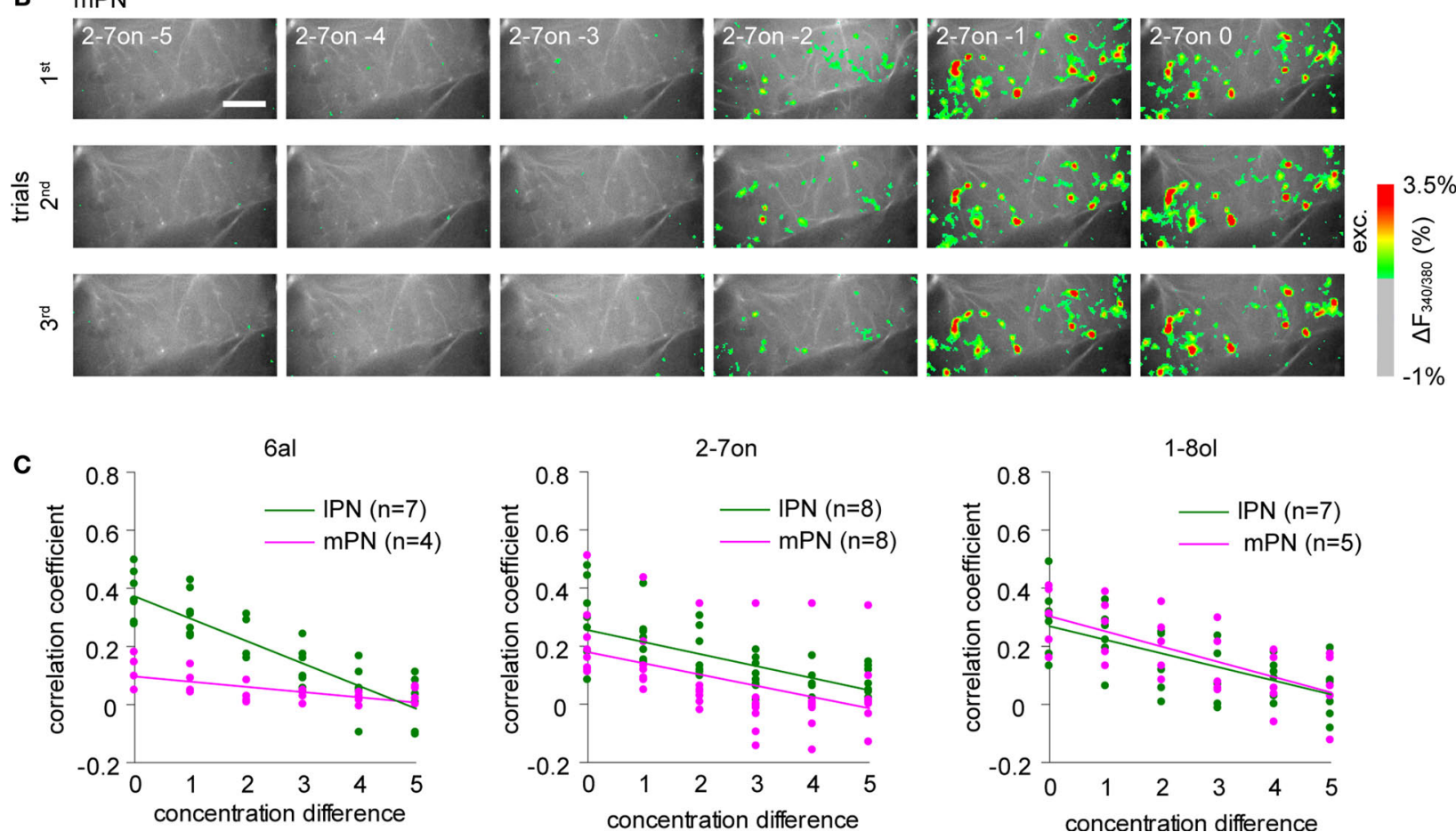

FIGURE 3 | Both IPN and mPN boutons encode odor concentration.

Changes of spatial activity patterns with increasing odor concentrations $\left(10^{-5}\right.$ to $10^{\circ}$, in six steps) in IPN and mPN bouton populations. Representative example of IPN bouton responses (A) and mPN bouton responses (B). Scale bars $=50 \mu \mathrm{m}$. (C) Response correlations between two concentrations plotted against their concentration differences. A value of zero in concentration difference indicates representation similarity in repetitions. Linear regression analyses revealed that response correlations are negatively correlated with increasing differences of concentrations for all odors both in IPN (green) and mPN boutons (magenta). Relations of this factor between IPN and mPN boutons differ between odors. The least-squares method was used. The following numbers of boutons were included in the analyses: IPN: 6al, hexanal, 540 boutons, 2-7on, 2-heptanone, 499 boutons, 1-8ol, 1-octanol, 538 boutons; mPN: 6al, 274 boutons, 2-7on, 667 boutons, $1-80 l, 282$ boutons. $n$ is the number of bees. activity patterns induced by the three odors at different concentrations was evaluated by calculating the linear correlation of the population responses (concatenated arrays of response traces from all boutons were used) and plotted against the concentration differences (6al, Figure 3C left; 2-7on, Figure 3C middle; 1-8ol, Figure 3C right). Linear regression analysis revealed that the response correlations and the concentration distances were negatively correlated in all three odors for both $\mathrm{PNN}$ and $\mathrm{mPN}$ boutons (lPN, 6al: $r=0.86, p<0.001,2-7$ on: $r=0.64, p<0.001,1-8 \mathrm{ol}$ : $r=0.65, p<0.001 ; \mathrm{mPN}, 6 \mathrm{al}: r=0.62, p<0.001,2-7$ on: $r=0.46$, $p<0.001,1-801: r=0.67, p<0.001)$. The similarity of responses depended on the difference in concentration. Larger concentration differences lead to lower similarity between responses in both IPN and $\mathrm{mPN}$ boutons. Response similarity also depended on the particular odor in both IPNs and mPNs, indicating that IPN and mPN boutons do not differ in the extent of similarity of their concentration representations.

However, as exemplified in Figures 3A,B, we often observed that $\mathrm{mPN}$ boutons showed clear dependence on rising concentrations of odors while IPN boutons did not (see also Figure S3A in Supplementary Material). Thus, we evaluated the strength of the bouton response at different concentrations by applying a linear regression analysis on the logarithm of these two values since a $\log$-log function describes the relationship between two factors as 
represented by a sigmoidal curve which is known as a fundamental relationship in receptor-ligand interactions at peripheral level (e.g. Hill equation). As shown in Figure 4A, boutons were statistically assigned to the positive dependency (left), no dependency (middle) and negative dependency (right) group.
Figure 4B shows the result for 2-7on. The majority of boutons in both $\mathrm{PN}$ and $\mathrm{mPNs}$ were categorized into the no dependence group (lPN 53.7\% in 499 boutons; mPN 68.7\% in 667 boutons), but more of IPN boutons fell into the negative dependence group (38.5\%) and more of $\mathrm{mPN}$ boutons fell into the positive dependence group

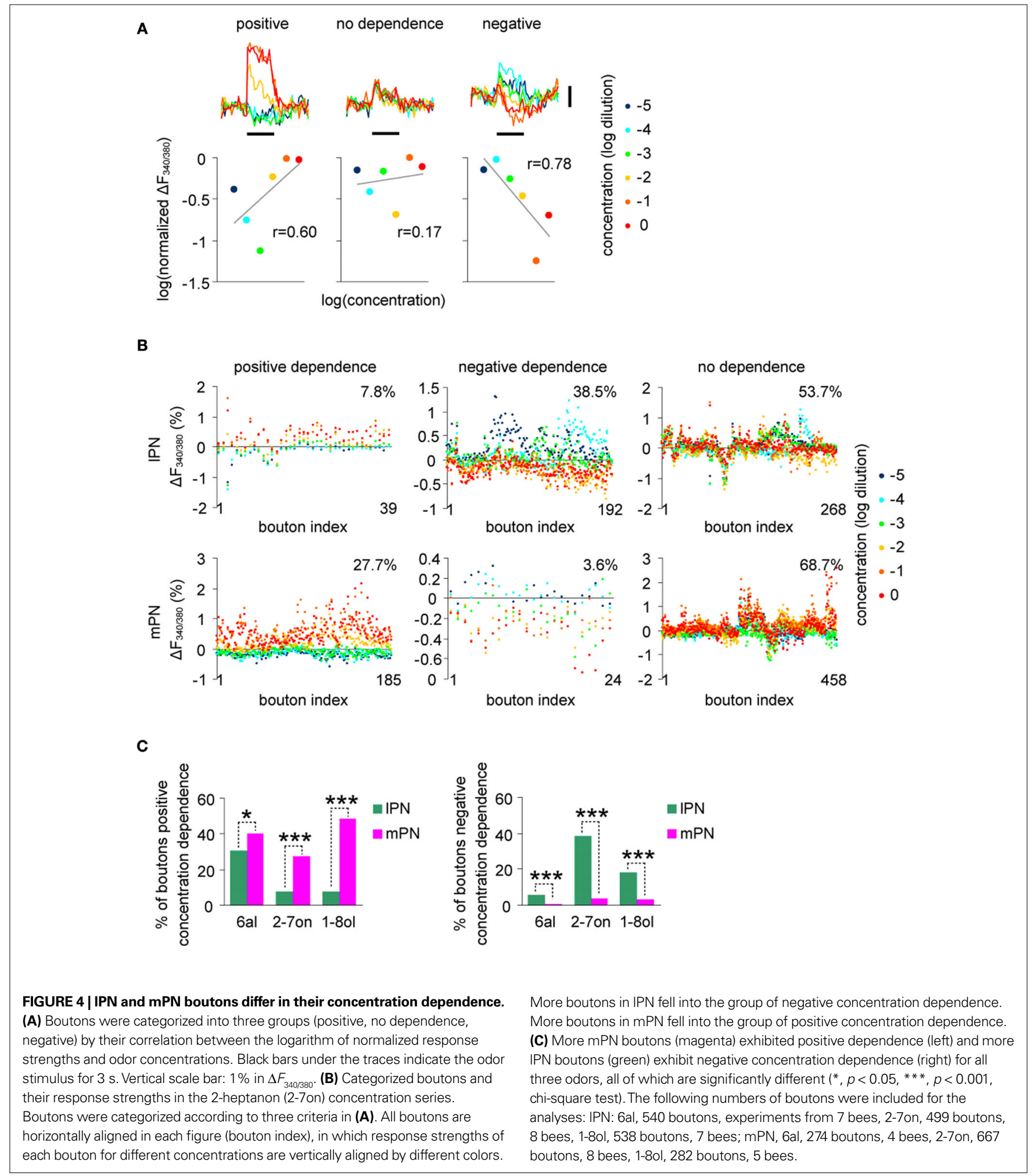


(27.7\%). The same result was observed for the other two odors, and the differences between IPN and mPN boutons were statistically significant for all three odors (Figure 4C, positive dependency, 6al: $p<0.05,2-7$ on: $p<0.001,1-8$ ol: $p<0.001$; negative dependency, 6al: $p<0.001,2-7$ on: $p<0.001,1-8$ ol: $p<0.001$; chi-square test), indicating a stronger concentration dependence in $\mathrm{mPN}$ boutons as compared to IPN boutons. The difference is partly reflected by the fact that more of IPN boutons were inhibited as odor concentration increased. This conclusion is also supported by the result of 3-way ANOVA applied for the number of responding boutons to variables of odor concentrations, tract types and odors (Figure S3A and Table S1 in Supplementary Material), which revealed a significant difference for a combinational effect of odor concentration and tract type (Concentration $\times$ Tract, $p=0.0019$ ). This implies that the effect of odor concentration on bouton responsiveness differs between IPN and mPN boutons.

\section{HIGHER ODOR SPECIFICITY AND SPARSER RESPONSES IN IPN BOUTONS AT HIGH ODOR CONCENTRATION}

Less positive concentration dependence of IPN bouton responses (Figure 4) might imply more odor-specific and sparser odor responses at higher concentrations. In order to assess this interpretation, we analyzed bouton responses to seven different odors at a dilution of $1 \%$, including the three odors which we used in the concentration experiments. 717 boutons $(71.7 \pm 5.8,10$ bees $)$ in IPNs and 708 boutons ( $88.5 \pm 7.2,8$ bees) in $\mathrm{mPNs}$ were taken into account.

Representative examples are shown in Figure 5A (IPN) and in Figure 5B ( $\mathrm{mPN})$. Again, stable odor-specific spatio-temporal response patterns were found. Stimulus repetitions were reliably represented in individual boutons, suggesting reliable $\mathrm{Ca}^{2+}$ measurements in these experiments (Figure S4B in Supplementary Material). When all boutons from different bees were taken into account, both IPN and mPN boutons responded to all odors applied (Figure S3B in Supplementary Material). Inhibitory responses were observed more often in IPN boutons than in mPN boutons.

Odor coding properties at the level of bouton populations were assessed (concatenated arrays of response traces from all boutons were used) by comparing the responses to repeated stimuli of two different odors (Figure 5C) (see Materials and Methods). A Kruskal-Wallis ANOVA revealed significant differences among the correlation coefficients within or between IPN and mPN boutons $(p<0.001)$. A post-hoc test (Steel-Dwass test, Dwass, 1960; Steel, $1960)$ showed significant differences between inter-trial correlations and inter-odor correlations of both tracts $(p<0.01)$. Odor coding in repetitions of the same odor is more reliable than that of two different odors in boutons of both tracts. The statistical analysis also revealed that inter-odor correlations between IPN and mPN neurons were significantly different $(p<0.05)$, while inter-trial correlations were not, indicating that odors were more distinctively represented in IPN boutons than in $\mathrm{mPN}$ boutons.

We further analyzed the differences in response-profiles between IPN and mPN boutons by calculating the probability of boutons responding with excitation or inhibition, applying threshold criteria for excitation and inhibition (see Experimental Procedures). As shown in Figures 5A,B, mPN responses were more likely to be excitatory and $\mathrm{IPN}$ responses were more likely to be inhibitory to odors. Except for 6al, the proportions of excitatory responses in $\mathrm{mPN}$ boutons and of inhibitory responses in IPN boutons were larger than the respective proportions in the other tract. Of the seven odors tested, $3.10 \pm 0.24$ odors elicited an excitatory response in lPN boutons ( $n=10$ bees), while $4.13 \pm 0.37$ odors caused excitation in $\mathrm{mPN}$ boutons ( $n=8$ bees) (Figure 5D). The distribution of effective odors causing excitatory responses is significantly different between IPN and mPN boutons (Number of effective odors $\times$ Tract, $p<0.005,2$-way ANOVA, IPN: $n=10$; mPN: $n=8)$. The average numbers of odors that elicited inhibitory responses were $5.04 \pm 0.23$ ( $n=10$ bees) in IPN boutons and $4.49 \pm 0.31 n=8$ bees) in mPN boutons (Figure 5E), and the difference was significant (Number of effective odors $\times$ Tract, $p<0.05,2$-way ANOVA, $1 \mathrm{PN}: n=10$; $\mathrm{mPN}$ : $n=8$ ). These results show that more $\mathrm{PPN}$ boutons responded with inhibition and more mPN boutons responded with excitation to odors at $1 \%$ concentration.

\section{MIXTURE SUPPRESSIVE EFFECTS ARE STRONGER IN IPN BOUTONS}

Next, we evaluated the effects of binary odor mixtures by comparing the mixture responses with those of the component responses. The following two categories of responses were defined (Figure 6B): (1) the response to the mixture is equal or higher than the response to the weaker component (additive mixture effect), and (2) the response to the mixture is lower than the response to the weaker response minus noise (suppressive mixture effect) (see Experimental Procedures). The additive group also includes mixture responses that lie between the responses to the weaker component and that of the stronger component. We include this response group because it is well possible that the two components compete at the level of the olfactory receptor molecule reducing the afferent input to mixtures. Two binary mixtures were tested: 2 -heptanone/1-octanol (2-7on/1-8ol) and hexanal/2-octanone (6al/2-8on). The analysis is based on the evaluation of 814 boutons ( $50.9 \pm 3.8,16$ bees) in the IPNs and of 444 boutons ( $63.4 \pm 6.3,7$ bees) in the mPNs.

Figure 6A exemplifies a suppressive mixture effect in IPN boutons and an additive mixture effect in $\mathrm{mPN}$ boutons in response to 6al, 2-octanone (2-8on) and their 1:1 mixture. In Figure 6B, the response traces of three representative boutons are shown. The first one (left) exhibited stronger responses to the binary mixture (red) while almost no responses were seen to its components (6al: black, 2-8on: cyan), indicating the addition of sub-threshold excitatory inputs from the 2 odors. The second response traces (middle) exhibited an intermediate response to the binary mixture, indicating averaging of a stronger excitatory response to one component and a weak inhibitory response to another component. As described above, we assigned these two cases to an additive mixture effect. The third response traces (right) exhibited an inhibitory response to the binary mixture and almost no responses to the components indicating inhibitory responses. We assigned this case to the suppressive mixture effect.

We calculated response strengths to $6 \mathrm{al}, 2-8$ on and their 1:1 mixture (Figure 6B), 2-7on, 1-8ol and their 1:1 mixture for all boutons. Suppressive mixture effects occurred in lPN boutons more often than in $\mathrm{mPN}$ boutons (Figure 6C, 2-7on/1-8ol, $p<0.001$, IPN: 10 bees, 485 boutons, mPN: 5 bees, 347 boutons; 6al/2-8on, $p<0.05$, lPN: 6 bees, 329 boutons, mPN: 2 bees, 97 boutons, loglikelihood test). 


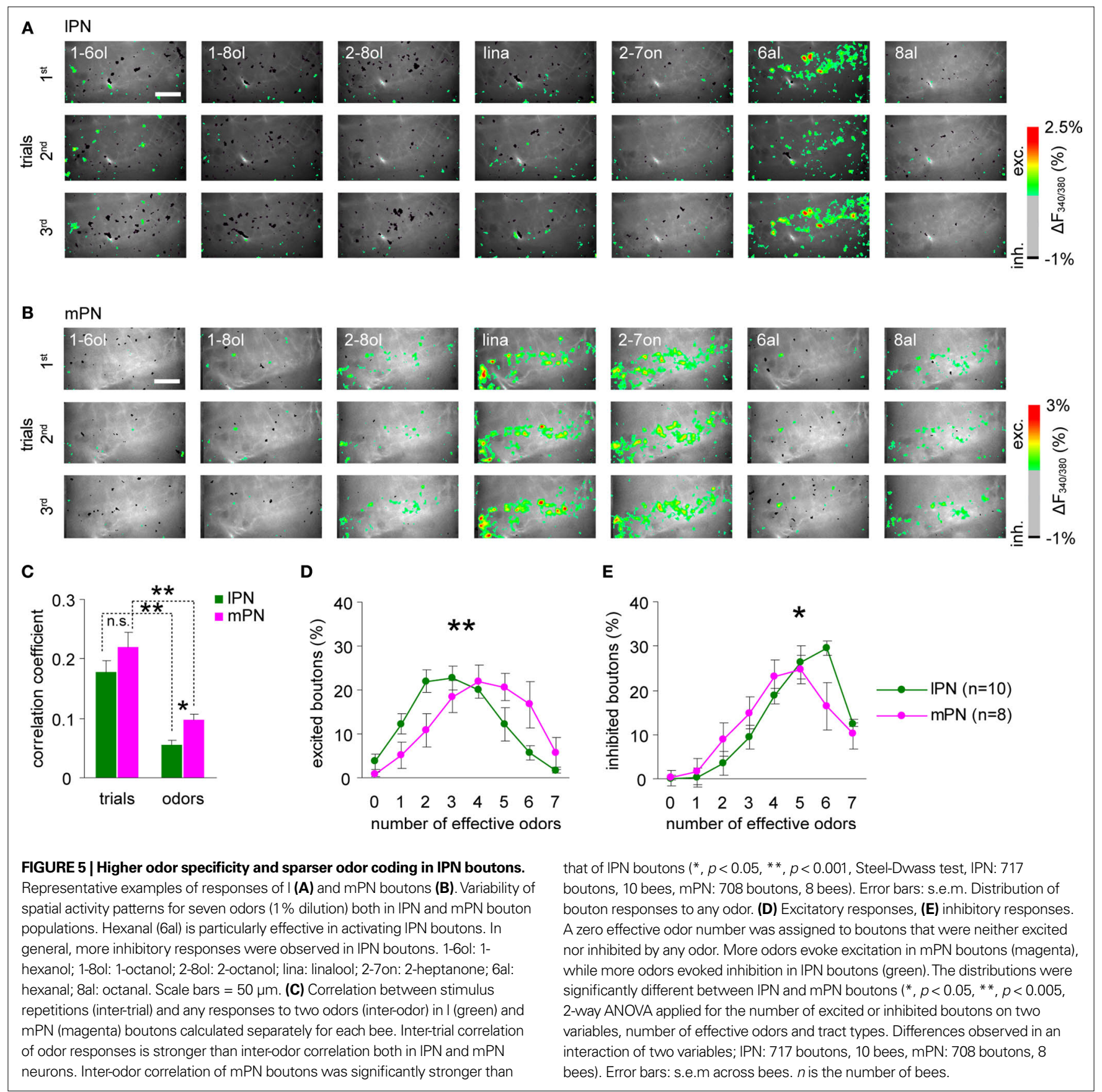

\section{DISCUSSION}

Olfactory systems for general environmental odors are characterized by parallel pathways but their functional separations are not well understood. We characterized odor response properties of axon terminal boutons of two anatomically distinct olfactory PN types in the honeybee brain, and found overlapping similarities and qualitative differences between them. First, the mPNs boutons often showed rising concentration dependence to a series of concentrations $\left(10^{-5}\right.$ to $10^{\circ}$ ) of three odors while IPN boutons were less concentration dependent. Second, at high concentrations IPNs were more narrowly tuned to odors and responded in a sparser way than mPNs. Third,
IPNs were more likely than mPNs to exhibit mixture suppression (antagonistic mixture coding). Thus odor representation in IPNs appears to be optimized for fine, relatively concentration-invariant odor discrimination and synthetic mixture processing. whereas odor representation in $\mathrm{mPN}$ are optimized for concentration coding and mixture processing combining the effects of the odor components (analytic mixture coding). These results suggest a functional division of odor processing in the two PN types.

It should be noted, however, that our results are based on a limited number of odors. It will be, therefore, necessary to test our hypothesis by using an extended number of odors. 

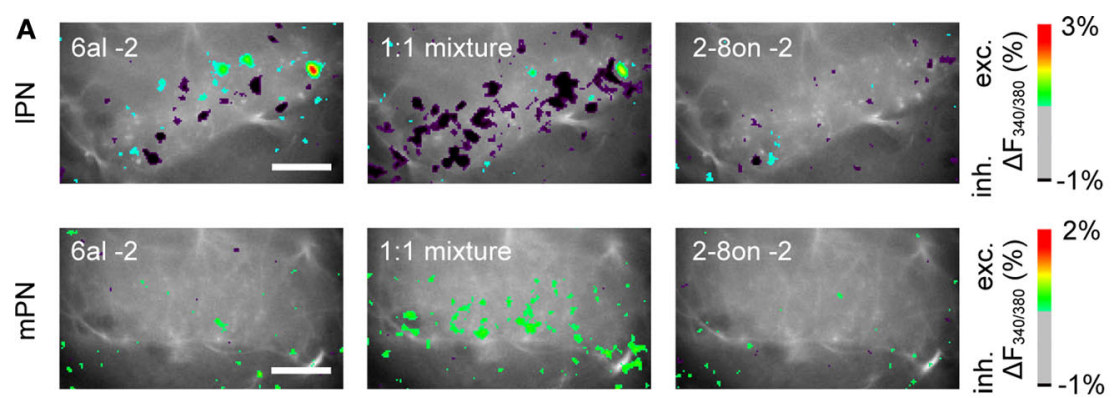

B
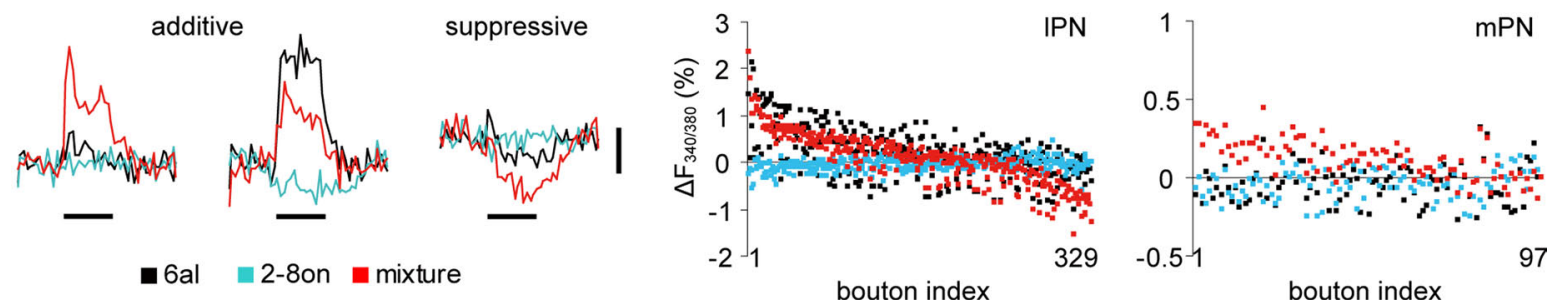

FIGURE 6 | Mixture suppression in IPN boutons. (A) Representative examples of IPN and mPN bouton responses to odor mixtures and their components. Shown are superimposed images of raw fluorescence images and trial-averaged (three trials) color-coded $\mathrm{Ca}^{2+}$ responses of boutons. In the IPN boutons, more inhibitory responses occurred with the $1: 1$ binary odor mixture of $1 \%$ hexanal (6al) and 1\% 2-octanon (2-8on). Meanwhile, more excitatory responses occurred with the 1:1 binary odor mixture in mPN boutons. Scale bars $=50 \mu \mathrm{m}$. (B) Boutons were categorized into two groups (additive or suppressive mixture effect) by the response strength to binary mixtures as compared to those of the components (see Experimental Procedure for details). All bouton responses were horizontally aligned in the right figures (bouton index), in which response strengths of each bouton for a binary mixture (red dots) and its components (6al: black, 2-8on: cyan) are vertically aligned by different colors. Substantial amount of IPN boutons ( $30 \%)$ exhibited minimum response strengths while mPN boutons mostly exhibited greater or equal response strength to a binary mixture compared to its components. Black bars under the traces indicate the odor stimulus for $3 \mathrm{~s}$. Vertical scale bar: $1 \%$ in $\Delta F_{340 / 380}$. (C) Percentage of boutons exhibiting the suppressive mixture effect. In both odor combinations, IPN boutons show greater proportions of mixture suppression than mPN boutons (27on/1-8ol, IPN: 485 boutons, 10 bees, mPN: 347 boutons, 5 bees; 6al/2-8on, IPN: 329 boutons, 6 bees, mPN: 97 boutons, 2 bees, ${ }^{*}, p<0.05,{ }^{* * *}, p<0.001$. loglikelihood test applied for absolute bouton numbers).

\section{TECHNICAL CONSIDERATIONS}

In this study, bouton responses exhibited relatively low correlation values (high variability) even for stimulus repetitions, which is in contrast to the AL glomeruli achieving very reliable odor responses (Joerges et al., 1997; Sachse and Galizia, 2002, 2003; Galizia and Kimmerle, 2004; Szyszka et al., 2005; Krofczik et al., 2009). The apparent 'lacking of reliability' in bouton responses may reflect a property of information processing at this stage with variability possibly resulting from inhibitory input via recurrent neurons of the mushroom body lobes (see below). The higher variability seen in our recordings may have also technical reasons. (i) Terminal boutons of PNs are very small, so their responses are relatively weak and spatially confined. Such small responding points can be easily affected by many measurement artifacts (such as very small movements). The small size does not allow much spatial averaging of activated pixels for noise reduction of time traces. It is obvious that noise will reduce correlation values across bouton responses. (ii) The sparseness of bouton responses. Often bouton responses were very specific to one or two odors within a series of odors. This property implies the necessity of including many of non-responding or weakly responding boutons in the correlation analysis. Thus the particular response property of boutons unavoidably reduces the correlation between boutons considerably.

Reduction of $\mathrm{Ca}^{2+}$ activity, which often characterized IPN bouton responses, may be explained by the following: a reduction in the number of arriving spontaneous or odor-induced action potentials, since the frequency of action potentials is mirrored in the $\mathrm{Ca}^{2+}$ dynamics of PNs at their input sites (Galizia and Kimmerle, 2004; Moreaux and Laurent, 2007). Odor-induced reduction of spike activity can result from both the inhibitory inputs within their respective glomeruli via GABAergic local interneurons in the AL (Sachse and Galizia, 2002) and from GABA-immunoreactive (ir) profiles onto boutons (Ganeshina and Menzel, 2001; Figure S2A in Supplementary Material), which relate to the recurrent axon branches of the protocerebral tract (PCT) feeding the output of the MB lobes back into the MB input (Gronenberg, 1987; Grünewald, 1999).

\section{GLOBAL GAIN CONTROL IN LPN BOUTONS}

We evaluated the effects of different odor concentrations in the range of $10^{-5}$ to $10^{0}$ in six steps for three odors (6al, 2-7on, 1-8ol) (Figures 3 and 4). In all three odors we found that both IPN and $\mathrm{mPN}$ boutons reacted to all concentrations (Figure S3A in Supplementary Material), though the response magnitude and dynamic depended on the particular odor and its concentration. At the population level of boutons in a particular bee, a measurement of response similarity was a reliable indicator of concentration differences (Figure 3C), indicating that both $\mathrm{PPN}$ and $\mathrm{mPN}$ boutons code concentration differences.

Concentration dependence of responses can be described by its relation to the logarithm of concentration applying a linear regression analysis (i.e. Hill equation, Figure 4). We found for both tracts 
that boutons may exhibit positive, negative and no concentration dependence. $\mathrm{mPN}$ boutons showed positive concentration dependence more frequently whereas IPN boutons showed positive and negative concentration dependence equally often indicating a rise in inhibitory input at higher concentrations.

The negative concentration dependence, i.e. reduction of response strengths with rising concentration leads to a decreasing dose response function in which response saturation occurs at intermediate concentrations. Sachse and Galizia (2003) showed that IPN glomeruli clearly exhibit rising concentration dependence. Since receptor-odor interactions must have a threshold above which the excitatory neural responses will rise, a decay in neural responses at even higher concentrations indicates rising inhibitory input at higher concentrations. Such an inhibitory input may reflect a global gain control. In crustaceans, a global inhibitory system has been proposed as a gain control to prevent saturation of odor-evoked responses and to allow encoding of odors over a wide dynamic range (Wachowiak et al., 2002). A similar argument was put forward to explain the odor mixture effects in the AL glomeruli of the honeybee (Joerges et al., 1997; Deisig et al., 2006). Both the $\mathrm{AL}$ and the MB calyx network can be involved in this process by GABAergic input, in the AL via local interneurons (Flanagan and Mercer, 1989), in the MB calyx via GABA-ir recurrent neurons (Gronenberg, 1987; Grünewald, 1999; Ganeshina and Menzel, 2001; Figure S2 in Supplementary Material). It is likely that the inhibitory input leading to the saturating and falling concentration dependence of boutons is localized in the output region of the PNs because Sachse and Galizia (2003) found rising concentration dependence of IPNs in the glomeruli. Different physiological characteristics between IPNs and mPNs could be responsible for the differing amounts of putative GABA inputs into the respective boutons. We are currently analyzing this possibility.

\section{OVERLAPPING RESPONSE-PROFILES BETWEEN IPN AND mPN BOUTONS}

PN boutons of both tracts responded to all seven odors applied at a constant concentration of $1 \%$, which corresponds to a rather high concentration under natural conditions. In both PN types, responses were more similar for the same than for different odors (Figure 5C), indicating odor identity coding in both tracts. The chemo-profiles of IPN and mPN boutons are highly overlapping (Figure S3B in Supplementary Material), which corroborates earlier findings (Sun et al., 1993; Abel et al., 2001; Müller et al., 2002). It is, therefore, unlikely that different categories of odors are encoded in separate tract neurons.

IPN boutons are characterized by sparser coding of odor identity (Figure 5) because a higher proportion of these boutons show a negative concentration dependence (Figure 4). This finding corresponds with an earlier study which found sparsening of IPN boutons' response profiles within the $\mathrm{MB}$ calyx possibly by presynaptic inhibitory inputs (Szyszka et al., 2005). Such a mechanism would explain the higher probability of inhibitory responses in IPN boutons. Thus, it is not surprising that our results do not match those of Müller et al. (2002), who determined broader chemo-profiles in IPN than in $\mathrm{mPN}$ using intracellular recording from the axon trunk. Our finding provides additional support to the conclusion presented above that global gain control mainly occurs at the MB calycal network.

\section{MIXTURE SUPPRESSION IN IPN BOUTONS}

Coding of mixtures is known to include non-linear processes at peripheral (Akers and Getz, 1993; Getz and Akers, 1995) and central sites (Tabor et al., 2004; Deisig et al., 2006; Silbering and Galizia, 2007). Interactions of components in mixtures can be synthetic, leading to difficulty in segmenting the perception of odor mixtures into their distinct components (Chandra and Smith, 1998; Wilson and Stevenson, 2003). The interactions can be synergistic or inhibitory (Tabor et al., 2004; Deisig et al., 2006; Silbering and Galizia, 2007), but the determination of synergistic interaction is problematic because additive concentration phenomena often counteract this effect (Duchamp-Viret et al., 2003). We found suppressive mixture effects, in which boutons decreased their responses to mixtures in comparison to the components.

Mixture suppression exists in both $\mathrm{IPN}$ and $\mathrm{mPN}$ boutons (Figure 6). However, more IPN boutons decreased their responses to binary mixtures than $\mathrm{mPN}$ boutons. Such inhibitory mixture interactions could result from inhibitory interactions between ORNs within the pore plates on the antennae (Akers and Getz, 1993; Getz and Akers, 1995), by the inhibitory network of the AL (Deisig et al., 2006) and/or by that of the MB calyx. Interestingly, this finding is in agreement with recent data from intracellular single cell recording and staining by Krofczik et al. (2009).

Inhibitory mixture interactions are thought to be a particularly important feature for encoding natural scents of flowers, because these odorants are composed of complex blends of many substances (Knudsen et al., 1993), and bees are not only able to discriminate between components and mixtures (Chandra and Smith, 1998; Müller et al., 2000; Deisig et al., 2001, 2002; Komischke et al., 2003), but also to extract the variability of proportions of components in mixtures across a large range of concentrations (Wright and Smith, 2004).

\section{PHYSIOLOGICAL CHARACTERISTICS OF IPN AND mPN BOUTONS IN A BIOLOGICAL CONTEXT}

Honeybees forage over long distances orienting to low concentrations of flower odors, which may suddenly change to high concentrations when the bee passes through an odor plume. In this phase, detecting concentration gradients could be more important than identifying an odor. It has been suggested that odor concentration is coded in the strength of excitation both in ORNs (Akers and Getz, 1993; Getz and Akers, 1994; de Bruyne et al., 2001) and PNs (Sachse and Galizia, 2003). Since we found that the strength of $\mathrm{mPN}$ boutons' excitatory activity was more concentration dependent than that of IPN boutons (Figure 4), the physiological characteristics of $\mathrm{mPN}$ boutons are thought to be particularly suitable in this behavioral context. By choosing between flowers and working within a flower, bees are exposed to high concentrations of odors. In this phase, odor identity is more informative for flower identification and learning. Indeed, bees achieve their best discrimination of odor identity at a high concentration (Getz and Smith, 1991; Bhagavan and Smith, 1997; Pelz et al., 1997). We found that IPN boutons exhibited sparser coding characteristics than $\mathrm{mPN}$ boutons in response to a high concentration (1\%) of odors, suggesting their physiology is suitable in this behavioral context. Categorizing foraging phases according to the distance of bees from the flower might 
be a component of context-specific behavioral tuning (Menzel, 1985). The MB may play an important role in the control of such behavioral switches (Mizunami et al., 1998; Menzel and Giurfa, 2001; Lachnit et al., 2004).

\section{ACKNOWLEDGMENTS}

We thank Mr. F. Schaupp, Mr. A. Behl and Dr. A. Galkin for technical assistance in data analyses, Ms. A. Klawitter for providing Ringer solution for $\mathrm{Ca}^{2+}$ imaging and technical assistance in immunostaining

\section{REFERENCES}

Abel, R., Rybak, J., and Menzel, R. (2001). Structure and response patterns of olfactory interneurons in the honeybee, Apis mellifera. J. Comp. Neurol. 437, 363-383.

Akers, R. P., and Getz, W. M. (1993). Response of olfactory receptor neurons in honeybees to odorants and their binary mixtures. J. Comp. Physiol. A 173, 169-185.

Bhagavan, S., and Smith, B. H. (1997). Olfactory conditioning in the honey bee, Apis mellifera: effects of odor intensity. Physiol. Behav. 61, 107-117.

Bozza, T., Vassalli, A., Fuss, S., Zhang, J. J., Weiland, B., Pacifico, R., Feinstein, P., and Mombaerts, P. (2009). Mapping of class I and class II odorant receptors to glomerular domains by two distinct types of olfactory sensory neurons in the mouse. Neuron 61, 220-233.

Chandra, S., and Smith, B. H. (1998). An analysis of synthetic processing of odor mixtures in the honeybee (Apis mellifera). J. Exp. Biol. 201, 3113-3121.

Chess, A., Simon, I., Cedar, H., and Axel, R. (1994).Allelic inactivation regulates olfactory receptor gene expression. Cell 78, 823-834.

Christensen, T. A., and Hildebrand, J. G. (2002). Pheromonal and host-odor processing in the insect antennal lobe: how different? Curr. Opin. Neurobiol. 12, 393-399.

de Bruyne, M., Foster, K., and Carlson, J. R. (2001). Odor coding in the Drosophila antenna. Neuron 30, 537-552.

Deisig, N., Giurfa, M., Lachnit, H., and Sandoz, J. C. (2006). Neural representation of olfactory mixtures in the honeybee antennal lobe. Eur. J. Neurosci. 24, 1161-1174.

Deisig, N., Lachnit, H., and Giurfa, M. (2002). The effect of similarity between elemental stimuli and compounds in olfactory patterning discriminations. Learn. Mem. 9, 112-121.

Deisig, N., Lachnit, H., Giurfa, M., and Hellstern, F. (2001). Configural olfactory learning in honeybees: negative and positive patterning discrimination. Learn. Mem. 8, 70-78.

Duchamp-Viret, P., Duchamp, A., and Chaput, M. A. (2003). Single olfactory sensory neurons simultaneously integrate the components of an odour mixture. Eur. J. Neurosci. 18, 2690-2696.

Dwass, M. (1960). Some k-Sample Rank-Order Tests. In Contributions to Probability and Statistics, I. Olkin, S. G. Ghurye, W. Hoeffding, W. G. Madow and H. B. Mann eds. (Stanford University Press), pp. 198-202.

Fishilevich, E., and Vosshall, L. B. (2005). Genetic and functional subdivision of the Drosophila antennal lobe. Curr. Biol. 15, 1548-1553.

Flanagan, D., and Mercer, A. R. (1989). Morphology and response characteristics of neurones in the deutocerebrum of the brain in the honeybee Apis mellifera. J. Comp. Physiol. A 164, 483-494.

Galizia, C. G., Joerges, J., Küttner, A., Faber, T., and Menzel, R. (1997). A semi-in-vivo preparation for optical recording of the insect brain. $J$. Neurosci. Methods. 76, 61-69.

Galizia, C. G., and Kimmerle, B. (2004). Physiological and morphological characterization of honeybee olfactory neurons combining electrophysiology, calcium imaging and confocal microscopy. J. Comp. Physiol. A 190, 21-38.

Ganeshina, O., and Menzel, R. (2001). GABA-immunoreactive neurons in the mushroom bodies of the honeybee: an electron microscopic study. J. Comp. Neurol. 437, 335-349.

Gao, Q., Yuan, B., and Chess, A. (2000). Convergent projections of Drosophila olfactory neurons to specific glomeruli in the antennal lobe. Nat. Neurosci. 3, 780-785.

Getz, W. M., and Akers, R. P. (1994). Honey bee olfactory sensilla behave as integrated processing units. Behav.

Getz, W. M., and Akers, R. P. (1995). Partitioning non-linearities in the response of honey bee olfactory receptor neurons to binary odors. BioSystems 34, 27-40.

Getz, W. M., and Smith, K. B. (1991). Olfactory perception in honeybees: concatenated and mixed odorant stimuli, concentration, and exposure effects. J. Comp. Physiol. A 169, 215-230. physiological properties of feedback Neural Biol. 61, 191-195.

Gronenberg, W. (1987). Anatomical and

and Dr. J. Rybak for technical advice on neural reconstructions. This study was supported by grants from the Deutscher Akademischer Austausch Dienst (DAAD) to N.Y. and from the Deutsche Forschungsgemeinschaft to R.M (Me 365-37).

\section{SUPPLEMENTARY MATERIAL}

The Supplementary Material for this article can be found online at http://www.frontiersin.org/systemsneuroscience/paper/10.3389/ neuro.06/016.2009/

neurons of the mushroom bodies in the bee brain. J. Exp. Biol. 46, $115-125$.

Grünewald, B. (1999). Morphology of feedback neurons in the mushroom body of the honeybee, Apis mellifera. J. Comp. Neurol. 404, 114-126.

Hallem, E. A., and Carlson, J. R. (2006). Coding of odors by a receptor repertoire. Cell 125, 143-160.

Homberg, U., Montague, R. A., and Hildebrand, J. G. (1988). Anatomy of antenno-cerebral pathways in the brain of the sphinx moth Manduca sexta. Cell Tissue Res. 254, 255-281.

Joerges, J., Küttner, A., Galizia, C. G., and Menzel, R. (1997). Representations of odours and odour mixtures visualized in the honeybee brain. Nature 387, 285-288.

Kirschner, S., Kleineidam, C. J., Zube, C. Rybak, J., Grünewald, B., and Rössler, W. (2006). Dual olfactory pathway in the honeybee, Apis mellifera. J. Comp. Neurol. 499, 933-952.

Knudsen, J. T., Tollsten, L., and Bergstrom, L. G. (1993). Floral scents - a checklist of volatile compounds isolated by head-space techniques. Phytochemistry 33, 253-280.

Kobayakawa, K., Kobayakawa, R., Matsumoto, H., Oka, Y., Imai, T., Ikawa, M., Okabe, M., Ikeda, T., Itohara, S., Kikusui, T., Mori, K., and Sakano, H. (2007). Innate versus learned odour processing in the mouse olfactory bulb. Nature 450, 503-508.

Komischke, B., Sandoz, J. C., Lachnit, H. and Giurfa, M. (2003). Non-elemental processing in olfactory discrimination tasks needs bilateral input in honeybees. Behav. Brain Res. 145, 135-143.

Krofczik, S., Menzel, R., and Nawrot, M. P. (2009). Rapid odor processing in the honeybee antennal lobe network. Front. Comput. Neurosci. 2, 9. doi: 10.3389/neuro.10.009.2008.

Lachnit, H., Giurfa, M., and Menzel, R. (2004). Odor processing in honeybees: Is the whole equal to, more than, or different from the sum of its parts? In Advances in the Study of Behavior, Vol. 34, P. J. B. Slater, J. S. Rosenblatt, C. Snowdon, T. Roper, H. J. Brockmann, and M. Naguib eds (Amsterdam, Elsevier), pp. 241-264.
Lei, H., Christensen, T.A., and Hildebrand, J. G. (2004). Spatial and temporal organization of ensemble representations for different odor classes in the moth antennal lobe. J. Neurosci. 24, 11108-11119.

Malun, D., Waldow, U., Kraus, D., and Boeckh, J. (1993). Connections between the deutocerebrum and the protocerebrum, and neuroanatomy of several classes of deutocerebral projection neurons in the brain of male Periplaneta americana. J. Comp. Neurol. 329, 143-162.

Menzel, R. (1985). Learning in honey bees in an ecological and behavioral context, In Experimental Behavioral Ecology and Sociobiology, B. Hölldobler and M. Lindauer, eds (Stuttgart, Gustav Fischer Verlag), pp. 55-74.

Menzel, R., and Giurfa, M. (2001). Cognitive architecture of a minibrain: the honeybee. Trends $\operatorname{Cog} n$. Sci. 5, 62-71.

Mizunami, M., Okada, R., Li, Y., and Strausfeld, N. J. (1998). Mushroom bodies of the cockroach: activity and identities of neurons recorded in freely moving animals. J. Comp. Neurol. 402, 501-519.

Mobbs, P. G. (1982). The brain of the honeybee Apis mellifera: the connections and spatial organization of the mushroom bodies. Philos. Trans. $R$. Soc. Lond. B 298, 309-354.

Mombaerts, P., Wang, F., Dulac, C., Chao, S. K., Nemes, A., Mendelsohn, M. Edmondson, J., and Axel, R. (1996). Visualizing an olfactory sensory map. Cell 87, 675-686.

Moreaux, L., and Laurent, G. (2007). Estimating firing rates from calcium signals in locust projection neurons in vivo. Front. Neural Circuits 1, 2. doi: 10.3389/neuro.04.002.2007.

Müller, D., Abel, R., Brandt, R., Zöckler, M., and Menzel, R. (2002). Differential parallel processing of olfactory information in the honeybee, Apis mellifera L. J. Comp. Physiol. A 188, 359-370.

Müller, D., Gerber, B., Hellstern, F., Hammer, M., and Menzel, R. (2000). Sensory preconditioning in honeybees. J. Exp. Biol. 203, 1351-1364.

Pelz, C., Gerber, B., and Menzel, R. (1997). Odorant intensity as a determinant for 
olfactory conditioning in honeybees: roles in discrimination, overshadowing and memory consolidation. J. Exp. Biol. 200, 837-847.

Rø, H., Müller, D., and Mustaparta, H. (2007). Anatomical organization of antennal lobe projection neurons in the moth Heliothis virescens. J. Comp. Neurol. 500, 658-675.

Sachse, S., and Galizia, C. G. (2002). Role of inhibition for temporal and spatial odor representation in olfactory output neurons: a calcium imaging study. J. Neurophysiol. 87, 1106-1117.

Sachse, S., and Galizia, C. G. (2003). The coding of odour-intensity in the honeybee antennal lobe: local computation optimizes odour representation. Eur. J. Neurosci. 18, 2119-2132.

Sandoz, J. C. (2006). Odour-evoked responses to queen pheromone components and to plant odours using optical imaging in the antennal lobe of the honey bee drone Apis mellifera L. J. Exp. Biol. 209, 3587-3598.

Sandoz, J. C., Deisig, N., de Brito Sanchez, M. G., and Giurfa, M. (2007). Understanding the logics of pheromone processing in the honeybee brain: from labeled-lines to across-fiber patterns. Front. Behav. Neurosci. 1, 5. doi: 10.3389/neuro.08.005.2007.
Silbering, A. F., and Galizia, C. G. (2007). Processing of odor mixtures in the Drosophila antennal lobe reveals both global inhibition and glomerulusspecific interactions. J. Neurosci. 27, 11966-11977.

Soucy, E. R., Albeanu, D. F., Fantana,A. L., Murthy, V.N., and Meister, M. (2009). Precision and diversity in an odor map on the olfactory bulb. Nat. Neurosci. 12, 210-220.

Steel, R. G. D. (1960). A rank sum test for comparing all pairs of treatments. Technometrics 2, 197-207.

Stocker, R. F., Lienhard, M. C., Borst, A., and Fischbach, K. F. (1990). Neuronal architecture of the antennal lobe in Drosophila melanogaster. Cell Tissue Res. 262, 9-34.

Sun, X. J., Fonta, C., and Masson, C. (1993). Odour quality processing by bee antennal lobe interneurones. Chem. Senses 18, 355-377.

Szyszka, P., Ditzen, M., Galkin, A., Galizia, C. G., and Menzel, R. (2005). Sparsening and temporal sharpening of olfactory representations in the honeybee mushroom bodies. J. Neurophysiol. 94, 3303-3313.

Tabor, R., Yaksi, E., Weislogel, J. M., and Friedrich, R. W. (2004). Processing of odor mixtures in the zebrafish olfactory bulb. J. Neurosci. 24, 6611-6620.

Vosshall, L. B., Wong, A. M., and Axel, R. (2000). An olfactory sensory map in the fly brain. Cell 102, 147-159.

Wachowiak, M., Cohen, L. B., and Ache, B.W. (2002). Presynaptic inhibition of olfactory receptor neurons in crustaceans. Microsc. Res. Tech. 58, 365-375.

Wilson, D. A., and Stevenson, R. J. (2003). The fundamental role of memory in olfactory perception. Trends Neurosci. 26, 243-247.

Wright, G. A., and Smith, B. H. (2004). Variation in complex olfactory stimuli and its influence on odour recognition. Proc. Biol. Sci. 271, 127-152.

Yoshihara, Y., Kawasaki, M., Tamada, A., Fujita, H., Hayashi, H., Kagamiyama, H., and Mori, K. (1997). OCAM: a new member of the neural cell adhesion molecule family related to zoneto-zone projection of olfactory and vomeronasal axons. J. Neurosci. 17, 5830-5842.

Zhang, X., Rogers, M., Tian, H., Zhang, X., Zou, D. J., Liu, J., Ma, M., Shepherd, G. M., and Firestein, S. J. (2004). Highthroughput microarray detection of olfactory receptor gene expression in the mouse. Proc. Natl. Acad. Sci. U.S.A. 101, 14168-14173.
Zube, C., Kleineidam, C. J., Kirschner, S., Neef, J., and Rössler, W. (2008). Organization of the olfactory pathway and odor processing in the antennal lobe of the ant Camponotus floridanus. J. Comp. Neurol. 506, 425-441.

Conflict of Interest Statement: The authors declare that the research was conducted in the absence of any commercial or financial relationships that could be construed as a potential conflict of interest.

Received: 28 July 2009; paper pending published: 25 October 2009; accepted: 17 November 2009; published online: 04 December 2009.

Citation: Yamagata N, Schmuker M, Szyszka $P$, Mizunami $M$ and Menzel $R$ (2009) Differential odor processing in two olfactory pathways in the honeybee. Front. Syst. Neurosci. 3:16. doi: 10.3389/neuro.06.016.2009

Copyright (c) 2009 Yamagata, Schmuker, Szyszka, Mizunami and Menzel. This is an open-access article subject to an exclusive license agreement between the authors and the Frontiers Research Foundation, which permits unrestricted use, distribution, and reproduction in any medium, provided the original authors and source are credited. 